\title{
Effects of acupuncture on foveation characteristics in congenital nystagmus
}

\author{
Tanya Blekher, Tetsuto Yamada, Robert D Yee, Larry A Abel
}

\begin{abstract}
Aims/background-To examine the effects of acupuncture at the sternocleidomastoid muscles on foveation characteristics in congenital nystagmus.

Methods-Six patients with congenital nystagmus (CN) received a series of treatments consisting of two needles inserted into each sternocleidomastoid, stimulated by tapping gently every 5 minutes, for 20 minutes per session. Their eye movements were recorded using scleral search coils and changes in their $\mathrm{CN}$ waveforms analysed at each point in the treatment. Changes in the stability and duration of foveation periods were examined.

Results-Four of the six patients showed improved foveation at the commencement of treatment; three maintained this response throughout the treatment period and after the needles were removed. In two, the CN waveform itself was modified. Conclusion-This study and others involving afferent stimulation to the neck and face suggest that projections from these areas to the reticular formation and vestibular nucleus may alter the behaviour of the pathophysiological mechanism underlying congenital nystagmus. (Br F Ophthalmol 1998;82:115-120)
\end{abstract}

Congenital nystagmus $(\mathrm{CN})$ is an ocular oscillation that usually appears in early infancy. It is most often, but not always, seen in association with visuosensory abnormalities. ${ }^{12}$ Thus, while a sensory abnormality may often be a precipitating factor in the appearance of $\mathrm{CN}$, it is not an obligate one. Although several models have been proposed, ${ }^{34}$ the origins of $\mathrm{CN}$ remain uncertain. A variety of distinctive waveforms have been described in recordings of the eye movements of $\mathrm{CN}$ patients. ${ }^{56}$ These may almost always be distinguished from the simpler waveforms seen in acquired nystagmus. One of the most common features of $\mathrm{CN}$ waveforms is the presence of segments of the slow phase wherein the eyes remain at or close to the point of regard with little or no movement. These foveation periods have been reported to enhance visual acuity. ${ }^{67}$

Numerous treatments have been described for CN. These include drugs, ${ }^{8}$ contact lenses, ${ }^{910}$ prisms, ${ }^{11}$ biofeedback, ${ }^{12}{ }^{13}$ acupuncture, ${ }^{14}$ and a variety of surgical procedures. ${ }^{15-18}$ Vibratory or electrical stimulation of the face and neck was found to improve foveation and acuity in some patients with $\mathrm{CN} .{ }^{19}$ In that study, the best predictor of visual acuity was duration of foveation. Interestingly, the more beneficial site of stimulation in this study was the same as in a previous investigation on the efficacy of acupuncture-the sternocleidomastoid muscles of the neck. The present study examines the effects of acupuncture treatment on foveation duration and stability in the same muscles in six patients with $\mathrm{CN}$.

\section{Methods}

After the procedures were explained and written informed consent was obtained, subjects were seated 1.0 metre from a screen onto which a red helium-neon laser spot 0.25 degree in diameter was rear projected. The subjects were asked to fix their eyes on the target as it stepped to plus or minus 10 degrees for calibration. Subjects were then presented with an illuminated Snellen chart in primary position and asked to focus on the large "E". Frequent reminders to keep looking at the target were given during the recording sessions to maintain alertness and the brevity of each individual recording trial minimised fatigue. Horizontal eye movements of the dominant eye were recorded using the scleral coil technique, ${ }^{2021}$ displayed on a rectilinear chart recorder and digitised at $1000 \mathrm{~Hz}$ with 12 bit resolution for off line analysis. Data were recorded at baseline before insertion of the needles, at 5 minute intervals thereafter for 20 minutes, and after the needles were removed. The length of each recording was about 30 seconds.

Six patients with horizontal congenital nystagmus (all males, ages ranging from 16 to 46 years) were studied. Because the waveform variability seen in congenital periodic alternating nystagmus can make evaluation of the efficacy of this or any other treatment difficult to determine, such patients were not included in this study. The method of stimulation was the same as described by Ishikawa et al. ${ }^{14}$ The stainless steel needle used for acupuncture was $40 \mathrm{~mm}$ in length, with a diameter of $0.2 \mathrm{~mm}$. During one session four needles were inserted within the muscle bellies of the sternocleidomastoid muscle about $10 \mathrm{~mm}$ through the skin at the distal and proximal points of the muscle insertion. The needles were inserted for a period of 20 minutes and then removed. Every 5 minutes the experimenter gently tapped the needles. These sessions were repeated several times at intervals of 2-3 weeks.

While ideally any study of a proposed treatment would best be done in a double blind fashion with a control group, no study of congenital nystagmus therapies to date, whether evaluating surgery, biofeedback, con- 


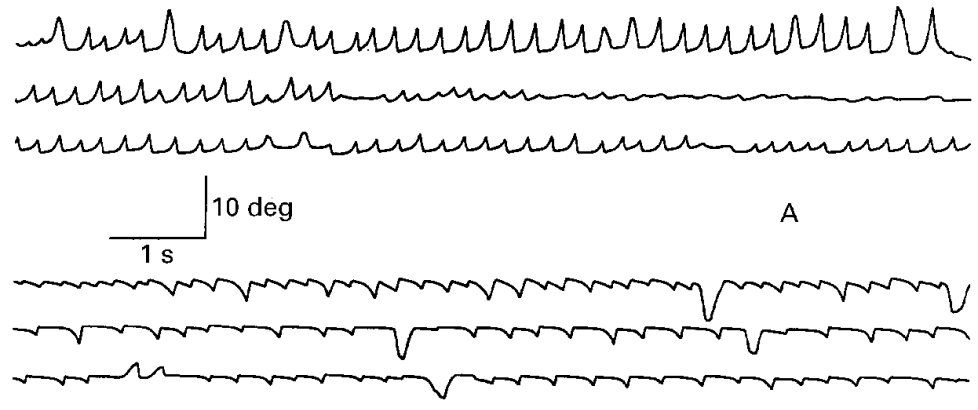

B
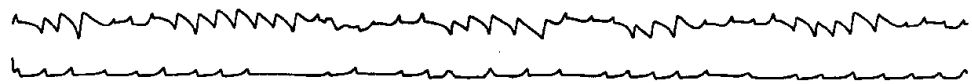

$\mathrm{C}$

Figure 1 Examples of $C N$ recorded in the three best responding patients: (A) TS, (B) $\mathcal{F P}$, and $(C)$ LH. For each patient, the top tracing was recorded before treatment, the middle after 20 minutes of acupuncture, and the bottom after the needles were removed. Calibration bars are 1 second and 10 degrees.

tact lenses, or neck vibration, has used this design. The present study is no exception. The issue is doubly complicated in the present instance both because it is unclear precisely what a control procedure would consist of and because of the strong influence which mental state of the patients has on their nystagmus, making the placebo effect even more powerful than usual. We did, however, run one sham treatment trial in our most responsive patient. Instead of inserting the needles at other locations (which could be criticised as still being an active treatment), the guide tubes were placed over the normal points of insertion and tapped at the same 5 minutes intervals as the needles normally were tapped. Given that the needles, when inserted, were barely noticeable, the patient's sensory experience was probably not much different from before.

For data analysis, statistical computation and graphical presentation, interactive pro-

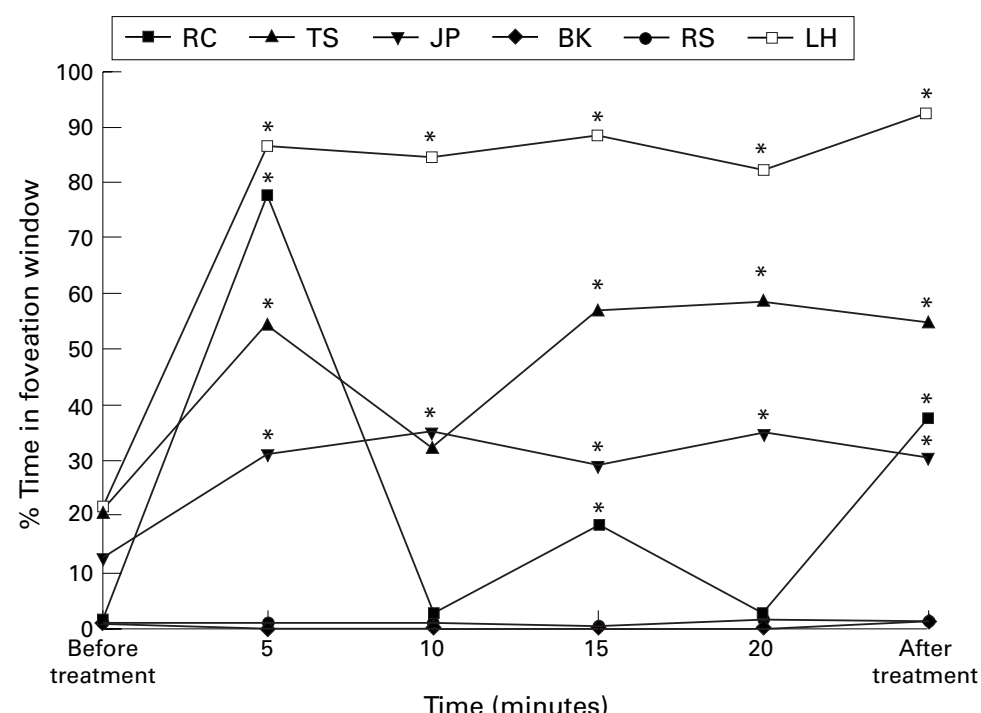

Figure 2 Percentage of time spent in the foveation window, whose criteria were that the eye was within 1 degree of the target and that slow phase velocity was $<4$ degrees/second. ${ }^{*} p<0.05$ from baseline. grams were written using Matlab and $\mathrm{C}++$. The digitised eye position signal was differentiated and the resulting velocity signal was filtered with a $50 \mathrm{~Hz}$ low pass filter. Data analysis of the signal was performed either in 5 second data segments, or over the entire 30 second recording period. Foveation periods were defined as those periods of the $\mathrm{CN}$ cycle which lay within a "foveation window", during which eye velocity was less than $4 \mathrm{deg} / \mathrm{s}$ and eye position was less than plus or minus 1 degree from the point of fixation. ${ }^{22}$ To calculate the average foveation time per record all points of the recording were analysed. To analyse the variance of foveation duration periods during a 30 second record, we divided each record into 1 or 2 second segments and calculated the empirical variances $\delta^{\prime}$ and $\delta^{\prime \prime}$. The $\delta^{\prime}$ was slightly less than $2 \delta$ " which implies the existence of memory in the signal. To avoid the influence of this memory we separated the 30 second signal into five intervals of 5 seconds in length with 1 second gaps between them. For statistical evaluation of foveation duration periods we used the distribution free multiple comparison procedure, based on the KruskalWallis rank sums test with significance at 0.05 level.

We also studied both the distribution of slow phase velocity and the mean slow phase velocity. To do this, we reconstructed the slow phase waveform without saccades. The desaccading algorithm was based on a combination of a Kalman filter ${ }^{23}$ and an algorithm for defining the fast phase threshold. Mean and standard deviation of slow velocity were calculated for each test condition and velocity distributions were built and compared. For statistical evaluation of mean slow velocity, Student's $t$ test was used with the significance at 0.05 level.

\section{Results}

\section{FOVEATION DURATION}

Of the six subjects tested, four demonstrated a significant increase in foveation duration ( $\mathrm{Tf}$ ) during and after acupuncture. Examples of the three most consistent responders are shown in Figure 1: patients (A) TS, (B) JP, and (C) LH, before the needles were inserted (top), after 20 minutes with needles in place (middle) and after the needles were removed (bottom). The percentage of time spent in the foveation window for all six patients is presented in Figure 2. Results are shown for recordings made at baseline, after 5, 10, 15, and 20 minutes of treatment and after the needles were removed. For four patients (RC, TS, JP, LH), Tf increased significantly after 5 minutes of acupuncture (Kruskal-Wallis test, $\mathrm{p}<0.05$ ). For patients JP and LH there was no significant difference in $\mathrm{Tf}$ after $5,10,15$, or 20 minutes of treatment and after needle removal; this was also true for TS at all but 10 minutes. For patient RC, $\mathrm{Tf}$ varied during and after acupuncture. Patients BK and RS showed no statistically significant changes in $\mathrm{Tf}$ at any time.

Because it is known that $\mathrm{CN}$ characteristics often depend on the mental state of the patients $^{36724}$ we wished to understand how 


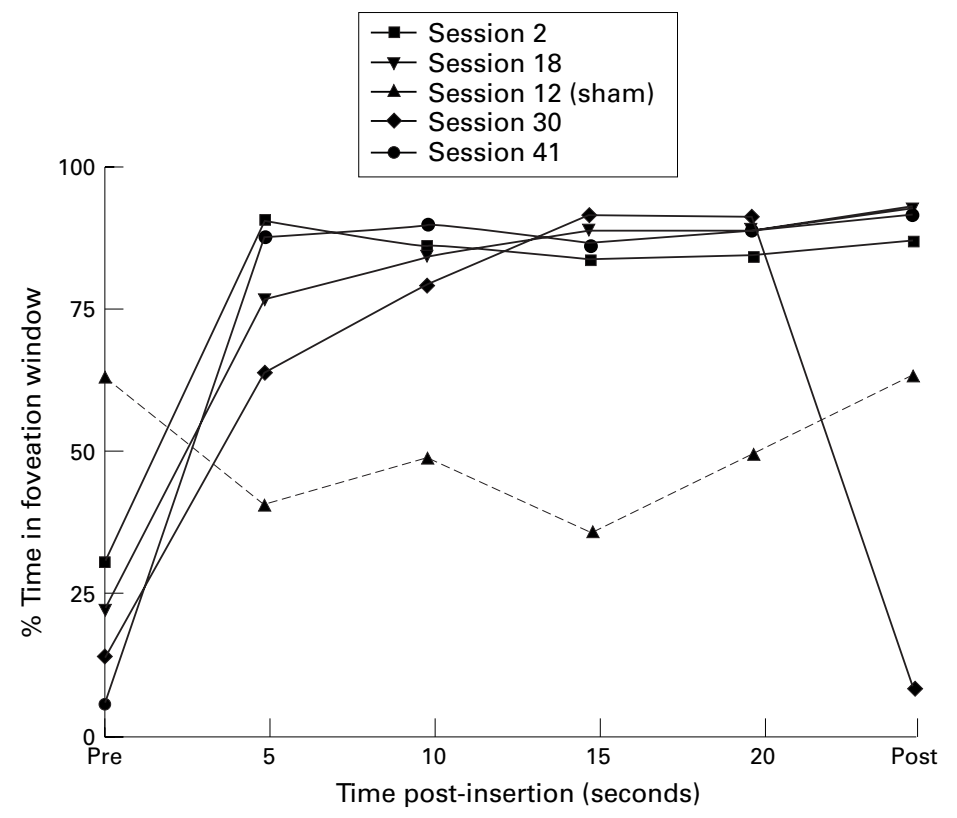

Figure 3 Percentage time in foveation window for patient $L H$ at four randomly selected sessions, plus the one "sham" treatment session. Treatment response was consistent, except for the "needles removed" recording at session 30, while the sham treatment appears to have, if anything, exacerbated the nystagmus.
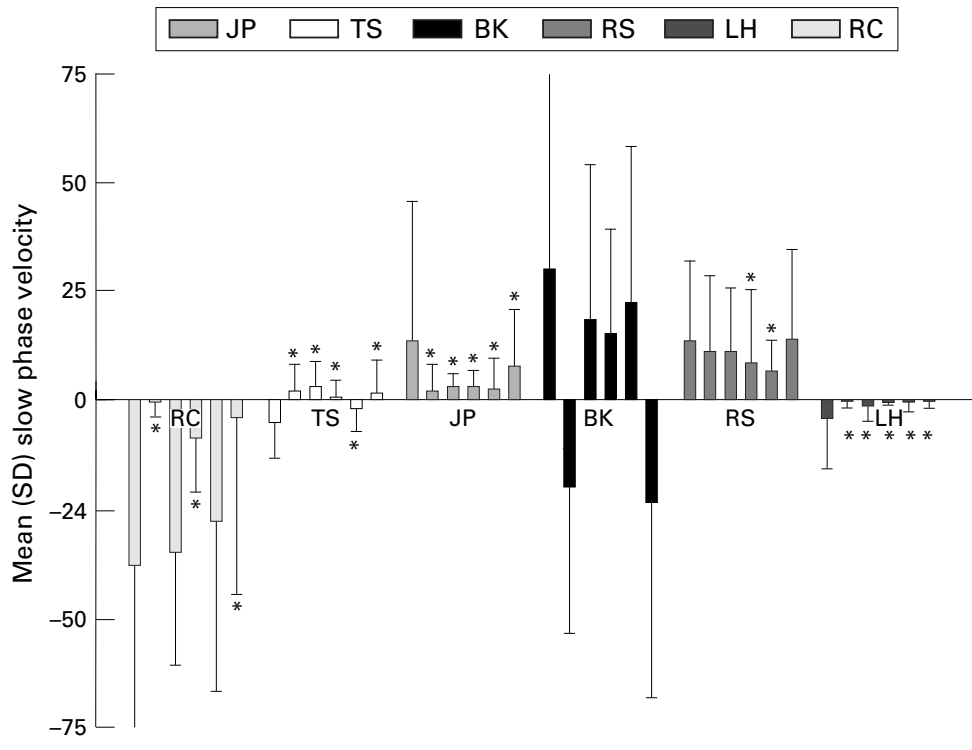

Figure 4 Mean (SD) slow phase velocity for all six patients at all six recording times. Asterisks indicate significant differences from baseline.

consistent the response to acupuncture was for the same patient across different sessions, weeks apart. An example of such a comparison is shown in Figure 3, which shows Tf for patient $\mathrm{LH}$ during randomly selected sessions $2,18,30$, and 41 and sham treatment session 16. Tf for each session is presented for baseline, every 5 minutes of treatment, and after removal of the needles. For all four treatment sessions Tf increased significantly during acupuncture and in three out of four sessions this improvement lasted after the needles were taken out. By contrast, the sham treatment session, conducted on a day when the patient's nystagmus happened to be relatively minimal to begin with, had the opposite effect, with foveation decreasing at each of the times when the guide tubes were tapped, as seen in Figure 3.
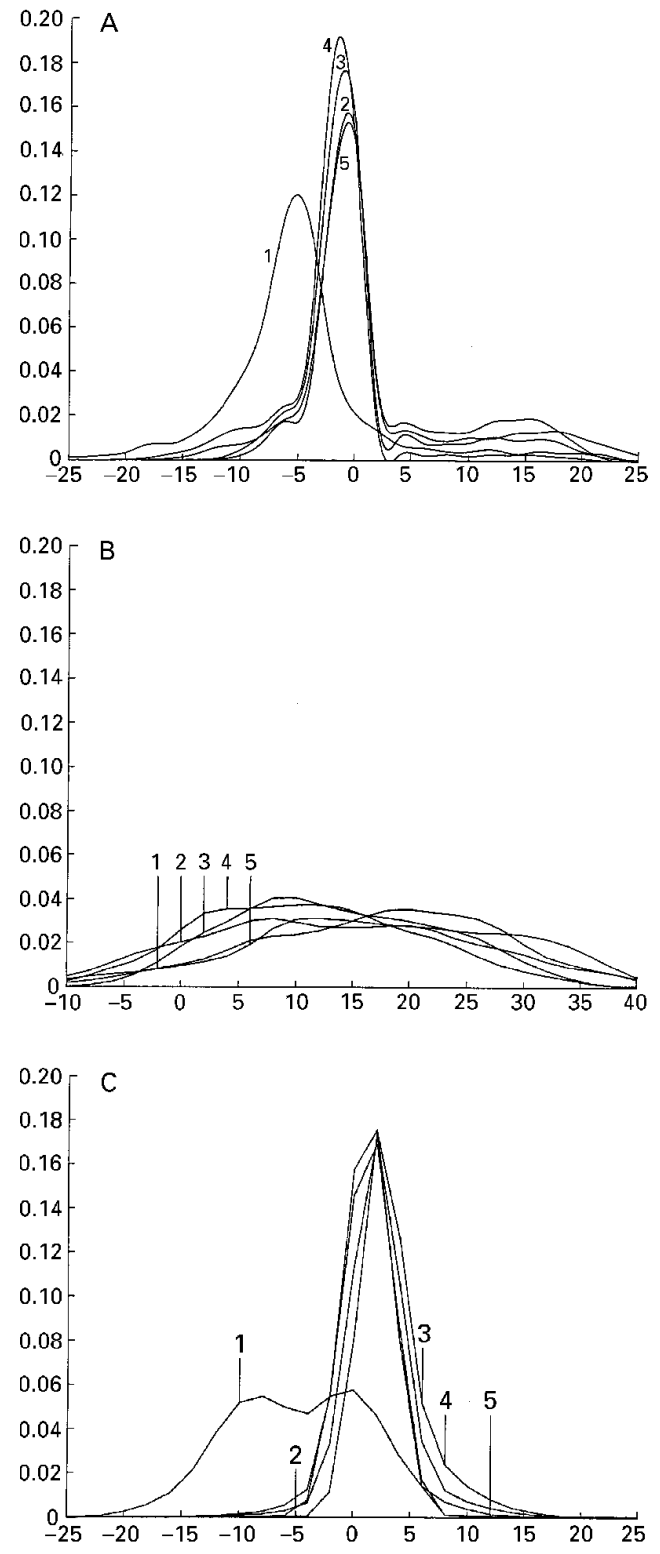

Figure 5 Slow phase velocity histograms for $(A)$ a good responder (fP), (B) a poor responder (BK), and (C) the good responder who showed a change from a bimodal to unimodal distribution $(L H)$. For $(A)-(C), 1=$ before treatment, $2=$ after 5 minutes, $3=$ after 10 minutes, $4=$ after 20 minutes, and $5=$ after removal of the needles.

In order to examine whether a "doseresponse" relation existed for this treatment, in one session with our most responsive patient (LH), we recorded his eye movements after each needle was inserted. Table 1 illustrates changes in $\mathrm{Tf}$ as needles were inserted first in the left upper position, second in right upper, third in left lower, and fourth in the right lower position. As we can see from this table, Tf was almost unchanged after insertion of the first and second needles but significantly increased after the third needle (Kruskal-Wallis test, $\mathrm{p}<$ 0.05). The fourth needle had almost no additional effect on Tf.

SLOW PHASE VELOCITY

After the slow phase of the primary position nystagmus was sampled over a period of 20 seconds, its velocity was analysed. The mean 


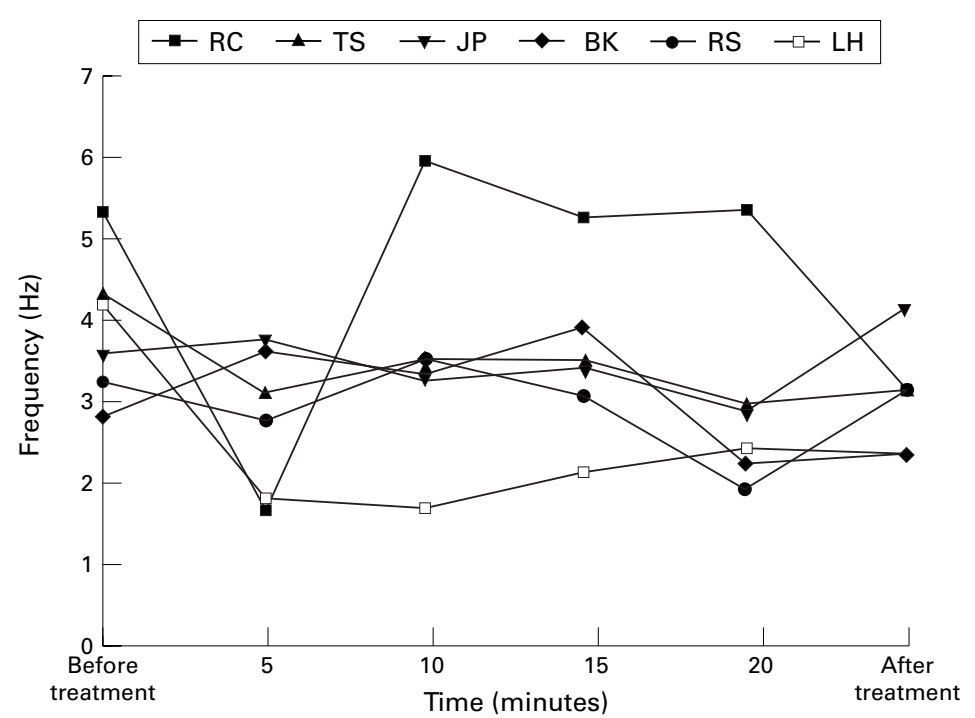

Figure 6 Nystagmus frequency over time.

and standard deviation of slow phase velocity are summarised in Figure 4 for the six patients at baseline, after $5,10,15$, and 20 minutes of treatment and after removal of the needles. All six patients demonstrated statistically significant reduction of mean slow phase velocity during acupuncture $(t$ test, $\mathrm{p}<0.05)$. Except for patient $\mathrm{RC}$, mean slow velocity was not significantly different in magnitude during acupuncture at $5,10,15$, and 20 minutes. The effect of removing the needles on the slow phase differed across patients. Patients RC and LH demonstrated further decreases in mean slow phase velocity, JP and RS demonstrated increases and BK and TS showed a change in sign but not in magnitude of mean slow phase velocity, implying that their nystagmus reversed direction but did not change otherwise. The decreasing and increasing of mean slow phase velocity correlated very well with changes in Tf for every patient.

The preceding comparison of means implicitly assumes that slow phase velocity is a normally distributed variable. Given the complex waveforms often seen in $\mathrm{CN}$, it was not clear that this assumption would necessarily hold. We therefore examined the slow phase velocity distributions for all subjects at the six testing points by constructing histograms of slow phase velocity, representative examples of which are shown in Figure 5. For five patients, distributions of slow phase velocity were unimodal before, during, and after acupuncture. During and after acupuncture the peak of the histogram shifted towards zero; one patient (LH) had a bimodal distribution before acupuncture, which became unimodal during and after treatment. This may be contrasted with the histograms for patient TS, for whom acupuncture caused a narrowing of the distri-

Table 1 Changes in foveation in patient $\mathrm{LH}$ as needles were sequentially inserted

\begin{tabular}{llllll}
\hline \multirow{5}{*}{ Before } & \multicolumn{5}{l}{ No of needles } \\
\cline { 2 - 6 } & 1 & 2 & 3 & 4 \\
\hline Tf & $16.0 \%$ & $14.6 \%$ & $21.3 \%$ & $75.5 \%$ & $81.6 \%$ \\
\hline
\end{tabular}

bution and a shift towards zero mean, but without changing the grossly Gaussian shape of the histogram.

In addition to the above changes, nystagmus frequency was relatively unchanged in four patients, decreasing with treatment in one (LH) and varying in another (RS) (Fig 6). For the group, there was a trend ( $t$ test, $\mathrm{p}=0.07$ ) for frequency to be lower after treatment than before.

\section{Discussion}

Our results are in very good agreement with and extend those reported by Ishikawa et al. ${ }^{14}$ In our study, four out of six patients demonstrated significant improvement of their nystagmus after acupuncture. We observed a trend towards a reduction in frequency and found decreased slow phase velocities, which led to significantly increased foveation duration periods. The improvement in foveation seen in these patients was observed at the 5 minute post-treatment measurement and was generally sustained, suggesting that it was not simply due to increasing fatigue or inattention. Examination of Figure 1 suggests that these effects may come about in more than one way. For TS, the nature of the $\mathrm{CN}$ waveform changes without a prominent change in amplitude; the increasing velocity exponential slow phase is altered in that longer foveation periods become prominent. For JP, the waveform does not change markedly but the nystagmus both becomes smaller and more sporadic. For LH, the jerk right beats virtually vanish and the remaining jerk left $\mathrm{CN}$ is greatly reduced. The changes in nystagmus waveforms are reflected in changes in the shape of the slow phase histograms in Figure 4. A simple scaling down of the oscillation would cause the histogram to become narrower, higher, and to shift towards the origin, but otherwise not to change in shape.

Speculation as to how acupuncture may affect $\mathrm{CN}$ is hindered by a lack of knowledge of both the basic mechanism of action of acupuncture and of the origins of $\mathrm{CN}$ itself. One of the hallmarks of $\mathrm{CN}$ is its labile nature. It may virtually stop with inattention or fatigue; conversely, visual effort directed in the dark towards an imagined target may exacerbate the nystagmus in the same fashion as does scrutiny of an actual object. ${ }^{67}$ Convergence and placement of the eyes at a particular gaze angle via a head turn frequently dampen the nystagmus. ${ }^{6}{ }^{18}{ }^{25-28}$ While gaze angle and viewing distance are readily fixed, internal variables such as attention and alertness are far harder to control. This may account for the absence of control treatments in other studies of $\mathrm{CN}$ therapies since it makes evaluation of them more difficult, in that the treatment variable may not be the only one changing. In the present instance, this difficulty is compounded by the lack of an obvious placebo treatment for acupuncture. The data in Table 1 , while not comparing acupuncture with placebo, appear to at least demonstrate a dose-response relation, in that as the four needles were inserted over a period of several minutes, an effect was 
only seen when the third needle was placed. Our sham treatment, which transiently stimulated the normal treatment points, also produced markedly different results from all the actual treatment sessions examined for this patient, appearing to exacerbate his nystagmus. Of course, difficulties in ruling out expectancy effects when evaluating treatments for $\mathrm{CN}$ are not unique to acupuncture.

The changes in waveform indicate that the nature of the underlying instability is affected by this treatment in some patients. The effects were generally similar to those seen when $\mathrm{CN}$ patients placed their eyes in their null position. They also closely resemble the positive results seen with afferent stimulation by Sheth et al. ${ }^{19}$ These investigators used either vibratory or electrical stimulation to the forehead or sternocleidomastoid muscle. They found that the best predictor of visual acuity was foveation duration, and that patients who responded positively to afferent stimulation showed an increase in $\mathrm{Tf}$, either with or without a reduction in amplitude. They also found that in some patients the standard deviation of foveation position decreased; this was not assessed in the present study. Negative responders in their study showed changes in the same variables, but in the opposite direction. In contrast, no patient in our study showed an exacerbation of nystagmus after acupuncture treatment. Sheth et al ${ }^{19}$ found vibratory, rather than electrical, stimulation of the neck muscles to be the combination of stimulating modality and site which most frequently yielded positive results. This would seem to be the combination closest to the acupuncture studies of Ishikawa et $a l^{14}$ and ourselves. Our studies further extend those by demonstrating a persistence of response in some patients after cessation of treatment. While acuity was not formally examined during and after treatment, the disappointment expressed by the better responding subjects when treatments had to be discontinued because of the departure of the author (TY) who was carrying them out is probably the best evidence that these patients did indeed perceive a sustained improvement in their vision.

One might not immediately expect somatosensory stimulation of the neck musculature to affect an ocular oscillation. Our results confirmed that afferent stimulation could markedly change the $\mathrm{CN}$ waveform. Several neural pathways exist which could be involved with these effects. Sensory information from the sternocleidomastoid travels at the level of C2 to the cervical plexus, whereas facial sensations are carried by the trigeminal nerve. ${ }^{29}$ Okuzawa $^{30}$ found that cervical stimulation modulated the vestibulo-ocular reflex (VOR) in cats, although there did not appear to be a direct projection from neck muscle afferents to the oculomotor nucleus, at least as evidenced from examining interactions between the effects of stimulating the cervical nerve and the branch of the third cranial nerve which innervates the inferior oblique.

Cervical modulation of the activity in the abducens nucleus induced by the VOR has also been reported. ${ }^{31}$ If these interactions are taking place at the level of the vestibular nucleus, then these projections could also be involved in modulation of the $\mathrm{CN}$ waveforms, given the role of the vestibular nuclei in neural integration and the potential relevance of abnormal functioning of this process to the pathogenesis of CN. ${ }^{3}$ If cervical afferents were stimulated by acupuncture and their activity tended to reduce the instability of the integrator presumed to contribute towards the development of $\mathrm{CN}$, then this could be one potential means whereby the beneficial effects of acupuncture could have occurred. Similarly, projections have also been noted from neck muscle afferents to the paramedian pontine reticular formation, ${ }^{32}$ a region usually thought of in conjunction with saccade generation. In the present context, this region may be more important for its role in the maintenance of arousal. Recalling that $\mathrm{CN}$ tends to diminish as the level of arousal decreases, perhaps the projections from the neck afferents serve to counteract the affects of arousal on the still unknown neural substrate responsible for $\mathrm{CN}$ but do so at a level below the centres of arousal per se. In this way, the nystagmus might be reduced in a way similar to that seen in less alert or sedated patients but without the necessity of producing somnolence to obtain an ocular motor benefit.

Patient LH, who was by far the best responder in this study, has already been discussed at length in the literature. ${ }^{22}$ His long standing history of $\mathrm{CN}$ was complicated when, after a transient loss of consciousness while doing sit ups, he developed intermittent oscillopsia. These periods of perceptual instability were found to correlate with the presence of periods of jerk right (JR) nystagmus. When jerk left (JL) nystagmus was present, he perceived the world as stable. Presumably, the JL nystagmus was his original waveform, with the JR beats having arisen from whatever ictal event occurred during exercise. Interestingly, both were unexceptional, exponentially increasing velocity jerk waveforms, but only the JL beats showed periods of foveation. Thus, the picture is not of an acquired nystagmus waveform superimposed on a pre-existing $\mathrm{CN}$; instead, it appears as if $\mathrm{LH}$ developed a new (for him) CN waveform to which his visual system had not adapted, thus leading to oscillopsia whenever it was present. Acupuncture treatment caused the disappearance of the $\mathrm{JR}$ beats and great reduction in the JL CN, abolishing the patient's oscillopsia. He reported that some benefit persisted for several weeks for distance viewing. It appears that the putative CNS insult and acupuncture both acted by modifying the behaviour of the pre-existing neural network giving rise to $\mathrm{CN}$. The other patients in the study, having uncomplicated histories of $\mathrm{CN}$, did not report oscillopsia. Clearly, advances in the understanding of both acupuncture and $\mathrm{CN}$ are needed before any of these speculations can be examined in greater detail. None the less, this study and others using afferent stimulation to treat $\mathrm{CN}^{14}{ }^{19}$ provide the first readily controlla- 
ble inputs to the CNS which have been shown to have a specific, beneficial effect on the nystagmus.

1 Gelbart SS, Hoyt CS. Congenital nystagmus: a clinical perspective in infancy. Graefes Arch Clin Exp Ophthalmol 1988;226:178-80.

2 Weiss AH, Biersdorf WR. Visual sensory disorders in congenital nystagmus. Ophthalmology 1989;96:517-23.

3 Tusa RJ, Zee DS, Hain TC, Simonsz HJ. Voluntary control of congenital nystagmus. Clin Vis Sci 1992;7:195-210.

4 Optican LM, Zee DS. A hypothetical explanation of congenital nystagmus. Biol Cybernetics 1984;50:119-34.

5 Dell'Osso LF, Daroff RB. Congenital nystagmus waveforms and foveation strategy. Doc Ophthalmol 1975;39:155-82.

6 Abadi RV, Dickinson CM. Waveform characteristics in congenital nystagmus. Doc Ophthalmol 1986;64:153-67.

7 Dell'Osso LF, Flynn JT, Daroff RB. Hereditary congenital Dell'Osso LF, Flynn JT, Daroff RB. Hereditary congenital
nystagmus: an intrafamilial study. Arch Ophthalmol 1974; 92:366-74.

8 Yee RD, Baloh RW, Honrubia V. Effect of baclofen on congenital nystagmus. In: Lennerstrand G, Zee DS, Keller EL eds. Functional basis of ocular motility disorders. Oxford: Pergamon, 1982:151-7.

9 Dell'Osso LF, Traccis S, Abel LA, Erzurum SI. Contact lenses and congenital nystagmus. Clin Vis Sci 1988;3:2289.

10 Abadi RV. Visual performance with contact lenses and congenit

11 Dell'Osso LF, Gauthier G, Liberman G, Stark L. Eye movement recordings as a diagnostic tool in a case of congenital nystagmus. Am f Optom Arch Am Acad Optom 1972;49:313 .

12 Abadi RV, Carden D, Simpson J. A new treatment for congenital nystagmus. Br f Ophthalmol 1980;64:2-6.

13 Ciuffreda KJ. Use of eye movement auditory biofeedback in the control of nystagmus. Am f Optom Physiol Optics 1982; 59:396-409.

14 Ishikawa S, Ozawa H, Fujiyama Y. Treatment of nystagmus by acupuncture. In: Ishikawa S, Mukuno K, Uga S, van Dalen JTW, eds. Highlights in neuro-ophthalmology. 6th ed. Amsterdam: Aeolus Press, 1987:227-32.

15 Helveston EM, Ellis FD, Plager DA. Large recession of the horizontal recti for treatment of nystagmus. Ophthalmology 1991;98:1302-5.
16 Fioretto M, Burtolo C, Fava GP. New surgical method for nystagmus without null point. Ophthalmologica 1991;203: $180-3$

17 Anderson JR. Causes and treatment of congenital eccentric nystagmus. Br F Ophthalmol 1953;37:267-81.

18 Abadi RV, Whittle J. Surgery and compensatory head postures in congenital nystagmus. A longitudinal study. Arch Ophthalmol 1992;110:632-5.

19 Sheth NV, Dell'Osso LF, Leigh RJ, Van Doren CL, Peckham HP. The effects of afferent stimulation on congenital nystagmus foveation periods. Vision Res 1995; 35:2371-82.

20 Robinson DA. A method of measuring eye movement using a scleral search coil in a magnetic field. IEEE Trans Biomed Eng 1963;BME-10:137-45.

21 Collewijn H, Van der Mark F, Jansen TC. Precise recording of human eye movements. Vision Res 1975;15:447-50.

22 Dell'Osso LF, Leigh RJ. Foveation period stability and oscillopsia suppression in congenital nystagmus. Neurooscillopsia suppression in
ophthalmol 1992;12:169-83.

23 Sauter D, Martin BJ, Di Renzo N, Vomscheid C. Analysis of eye tracking movements using innovations generated by a

24 Abel LA, Williams IM, Levi L. Intermittent oscillopsia in a case of congenital nystagmus: Dependence upon waveform. Invest Ophthalmol Vis Sci 1991;32:3104-8.

25 Dickinson CM. The elucidation and use of the effect of near fixation in congenital nystagmus. Ophthal Physiol Optics 1986;6:303-11.

26 Dell'Osso LF, Flynn JT. Congenital nystagmus surgery: a quantitative evaluation of the effects. Arch Ophthalmol 1979;97:462-9.

27 Pratt-Johnson JA. Results of surgery to modify the null-zone position in congenital nystagmus. Can f Ophthalmol 1991; 26:219-23.

28 Ukwade MT, Bedell HE. Variation of congenital nystagmus with viewing distance. Optom Vis Sci 1992;69:976-85.

29 Berkowitz BKB, Moxham BJ. A textbook of head and neck anatomy. London: Wolfe Medical Publishers, 1988.

30 Okuzawa I. Integrative action of vestibular and neck afferent projections to the oculomotor nuclei in the cat. $\mathscr{f p n} \mathcal{F} O \mathrm{ph}$ thalmol 1981;25:18-27.

31 Hikosaka O, Maeda M. Cervical effects on abduscens motoneuron and their interaction with vestibulo-ocular reflex. Exp Brain Res 1973;18:512-30.

32 Buettner-Ennever JA, Buettner U. The reticular formation. In: Buettner-Ennever JA, ed. Neuroanatomy of the oculomotor system. Amsterdam: Elsevier, 1988:119-76. 